\title{
A practical approach to diagnosing and managing advanced recurrent head and neck cancer
}

Volume 7 Issue 6 - 2017

Keywords: diagnosing, head and neck cancer, squamous cell cancers

\section{Introduction}

In today's literature, there are very few accounts, whether in textbooks or published literatures, that systematically describe the challenges and approaches to managing recurrent head and neck cancer.

Recurrent head and neck cancer is this difficult entity to diagnose and manage. This group of pathologies entails a large variety of tumor types, behavior which is not always predictable and is very variable clinical presentation course based on tumor characteristics, location, patient factors and condition and previous treatments. This paper aims to describe the different types of recurrence and the diagnostic approach as well as treatment options including supportive therapy. Literature regarding this topic is not extensive considering the fact that a significant number of squamous cell cancers of the head and neck recur. This is also rarely covered in conferences and major medical meetings as well. Head and neck cancer recurrence can be quite devastating to the patients and their families. Around 50 to $60 \%$ of stage III and IV head and neck cancers recur, which makes it more or less common condition faced by head and neck oncologic specialists. ${ }^{1,2}$ The survival rate ranges between 15 and $40 \%$. Survival time after local and regional recurrence is 6 to 9 months and $60 \%$ are due to local and regional recurrence. ${ }^{3,4} 90 \%$ of the patients with metastases die from uncontrolled disease on the primary site or the neck. $^{4}$

Old recurrent head and neck cancers present at an advanced stage with poor prognosis. As an example, recurrent early laryngeal cancers such as T1 and T2 vocal fold squamous cell carcinomas have a favorable survival rate if discovered early and treated appropriately (85-95\%). More advanced cancers of the larynx, recurrent oropharyngeal cancers, nasopharyngeal and hypopharyngeal cancers have a lower survival rate.

The goals of treatment include the following:

1. Cure

2. Prolongation of life (with good quality of life)

3. Reduction of local and regional disease, and alleviation of symptoms

\section{Palliation of pain and control of bleeding.}

Figure 1, the modality of choice for treating recurrent head and neck squamous cell carcinoma is usually complete surgical resection. The decision to operate on patients with advanced complex tumors requires thorough planning in the context of a multidisciplinary medical team that includes otolaryngologists, reconstructive surgeons, medical oncologists, radiation oncologists, speech/swallowing therapists, psychologists, pathologists, radiologists and others. In this
Alain Sabri
Otolaryngology Head and Neck Surgery, Lebanon

Correspondence: Alain Sabri, Otolaryngology-Head and Neck Surgery, Lebanese American University, Rizk Hospital, Beirut, Lebanon,Tel+96I-3-I54 I55, Email alainsabri@yahoo.com

Received: September 06, 2016 | Published: July 05, 2017

decision analysis tree, the first question that we have to ask ourselves is when to say no. It is not recommended to operate on patients with the following:

a. Multiple distant metastases (unresectable), or even a single metastasis that is advanced or an unfavorable location

b. Fixed disease after prior treatment (prior surgery, chemotherapy and/or radiation therapy)

c. Intratemporal fossa, parapharyngeal space cancers involving the skull-base (relative contraindication)

d. Supraclavicular disease after treatment (relative contraindication)

e. Skin metastases (by experience, patients with multiple skin metastasis have a very low rate of cure)

f. Significant comorbidity such as advanced cardiovascular disease and poor performance status

g. Advanced grade and extent of recurrent cancer after prior treatment.

One study that is significant is from the Mayo Clinic which concluded that there were no long time survivors in patients with recurrent neck disease after neck dissection regardless of subsequent treatment.

\section{Patient evaluation}

A good patient evaluation, as with every field in medicine, includes a thorough history including collection of previous pathology slides, operative reports, details of prior treatment and a study of risk factors. A complete physical examination including palpation of the previously treated tumor location and looking for subtle bleeding at the palpated area can be very helpful in detecting early hidden recurrences at sites such as the tongue base. Systematic examination of cranial nerves in addition to a full head and neck examination and fiber optic naso-pharyngolaryngoscopy is performed. Laboratory studies include disease-specific lab work in addition to the general hematology and chemistry panels. Radiologic studies include a CT scan, MRI, PET/CT, which are critical in evaluating recurrent cancer, in addition to angiography in cases of vascular involvement. Once the patient is fully and thoroughly evaluated, the patient is restaged and it is in fact, the stage of recurrent cancer that determines survival more than the original TNM. 


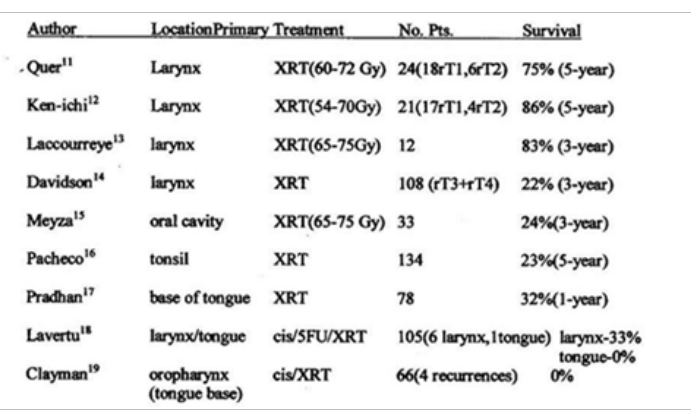

Figure I The modality of choice for treating recurrent head and neck squamous cell carcinoma is usually complete surgical resection.

\section{Treatment of recurrent head and neck squamous-cell carcinoma}

The following are the treatment options for recurrent head and neck squamous cell carcinoma:

Surgery at the primary site, neck dissection, reconstruction and (rarely) surgery for metastases: This is the cornerstone of treatment for cure in recurrent head and neck cancer that usually follows prior surgery, chemotherapy, radiation therapy or any combination of these therapeutic modalities. The literature has demonstrated that surgical salvage yields the best results. In a study by Goodwin, et al in a large number of patients has found that for recurrent stage I, there is a $73 \%$ survival at 2years; recurrent stage II $-67 \%$, recurrent stage II $-33 \%$, and recurrent stage IV $-22 \%$. This was based on an analysis of 32 published reports. ${ }^{1,5}$ The study concluded that survival depends on the stage of the recurrence and not on the original stage, and the location of the primary plays a major role in the success of the salvage. Evidently, the tongue base and the pharynx are the most difficult sites to salvage. Surgery for earlier laryngeal cancer such as T-1 lesions yields a disease-free survival of over $90 \%$ and for T- 2 ranging between $80-90 \%$. These are usually managed by endoscopic LASER partial laryngectomies including cordectomies, supraglottic laryngectomies and other endoscopic procedures. Open laryngeal surgeries still have a role today in the management of recurrent cancers in the form of supraglottic, fronto-lateral, hemi-laryngectomies, or supra-cricoid laryngectomies with good swallowing and acceptable voice results. ${ }^{6,7}$

Radiation therapy (conventional, IMRT, Stereotactic radio surgery, and others): Stereotactic radio surgery for locally recurrent head and neck cancers or skull-base metastasis has been performed. ${ }^{8,9}$ Studies published by Cmelak, Chua, Buatti and Firlik revealed different local control rates, ranging between 33 and $100 \% .{ }^{10-13}$ This is a very good option for local control and it may be curative. This has been used mostly in recurrent nasopharyngeal cancers and skull base localized lesions.

Chemotherapy: It has evolved greatly in the head and neck care which started with Methotrexate in the 1960s, all the way to the Carboplatin and Paclitaxel in the 80's and 90's and now there are evolving therapies involving Cetuximab - a monoclonal antibody. The chemo-radiotherapy for locally recurrent head and neck cancers also yields a survival rate ranging from 15 to $50 \%$. Platin-based chemotherapy alone has a response rate of around 30\%. New studies by Vermokin et al and others have shown that adding Cetuximab to Platinum and Fluorouracil improves the overall survival and almost doubles the response rate, which is feasible with acceptable side effects. ${ }^{14-18}$ It significantly reduces pain and swallowing problems and has no negative effect on the quality of life. This is the regimen that is recommended for recurrent head and neck squamous cell carcinoma which is not respectable.

Combination of the above: For selected cases, resection followed by chemotherapy and radiation is a tailored treatment of choice. These are performed for recurrent extensive disease and have shown acceptable survival rates in well selected patients.

Experimental Protocols - There are other novel target agents in squamous cell carcinoma of the head and neck including the following: ${ }^{18-20}$

i. Vascular EGF and VEGF receptor (anti-angiogenesis)

ii. Integrin inhibitors

iii. Histone deacetylase inhibitors which affect the DNA

iv. SRC inhibitors (intra-cellular Tyrosine Kinase)

v. Proteasome inhibitors

vi. IGFR inhibitors

Note: There are no Phase III trials on the above.

\section{Surgical treatment of distant metastasis}

There are no definitive large scale controlled studies on the benefits of treating distant metastases. Many small series and case reports have been published demonstrating the benefits of metastatectomies. ${ }^{15}$ In a healthy patient who has a single lung metastasis, most treating physicians decide to perform lung resection, and treat the primary site as well for the best chance of cure. This depends on patients overall status, and situation of the loco-regional disease. Many patients survived 'metastatectomies' and primary treatment in cases published in the literature and in unpublished report and physician experience.

\section{Head and neck reconstruction}

Head and neck reconstruction has grown greatly over the past three decades with the development and advancements of microvascular free flap reconstruction. The reconstructive ladder in general begins with healing by secondary intension (granulation tissue) followed by primary closure, skin grafts, local flaps, regional flaps, and microvascular free flaps. ${ }^{21-30}$ In recurrent head and neck cancer surgery, the resulting defect is usually large and complex. In addition, many of these patients have received radiation, so the quality of the tissues in the surgical bed is poor with an increased chance of infections, fistulas and breakdown. These patients usually require reconstruction with regional flaps including the deltopectoral (Bakamjian) and the pectoralis major flaps, which are the workhorses of head and neck reconstruction. Microvascular osteo-cutaneous reconstructions include the scapular and fibula free flaps for mandibular or maxillary reconstruction. ${ }^{31,32}$ For soft tissue reconstruction such as tongue, oropharynx and pharynx: Forearm free flap (Chinese flap), lateral thigh, lateral arm, rectus abdominis and latissimus dorsi free flaps. ${ }^{33-35}$

It is extremely important that two teams work together simultaneously: the primary resection team and the reconstructive team. This shortens the operating room time significantly and helps each team focus on the task at hand. ${ }^{36-40}$ Wider margins are usually taken so that the chance of recurrence is minimized. After the generous resection with wide margins, the defects are usually large and the reconstructive team is responsible for the flap harvest and reconstruction that yields the best functional and aesthetic results while covering the large vessels to avoid vascular events such as carotid blowouts. Soft tissue is replaced with soft tissue and bone is 
reconstituted with bone. Occasionally, sensate free flaps are part of the reconstructive armamentarium to restore sensation in cases of oral, pharyngeal or oropharyngeal reconstruction to improve swallowing and avoid aspiration. The flap nerve (such as the lateral ante-brachial cutaneous nerve in the forearm flap) is anastomosed to the buccal or lingual nerves to reconstitute sensation in the reconstructed tissues (Figure 2). ${ }^{16}$

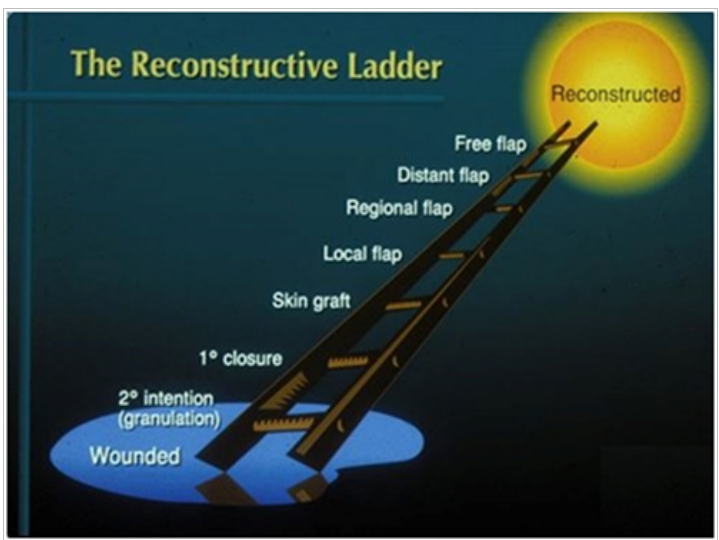

Figure 2 Reconstructive ladder.

\section{Palliative care}

Recurrent head and neck cancers can be a highly devastating disease. There are times, where no options are available in terms of treatment for cure and the medical team has to communicate honestly and openly with the patient and the family to provide options for palliative care including pain control, psychological support and other soothing measures. ${ }^{40-47}$ The pain and hospice teams as well as psychologists/psychiatrists are consulted early on to assist in are alleviating the patients' and families' suffering. ${ }^{21}$

\section{Conclusion}

Primary head and neck cancer is not an uncommon disease entity that physicians of all specialties encounter. Recurrent head and neck cancer of all stages is commonly seen, especially in tertiary care Medical Centers. The management options are discussed and the treatment strategy depends on the site, recurrent tumor stage, patient's health status, local tissue factors, extent of tumor and types of prior therapy which may include surgery, radiation, chemotherapy, other therapies or any combination of these modalities.

Discussion and studies are discussed in the literature and in conferences hopefully this trend will continue to develop. Overall, the mainstay of treatment for the majority of recurrent squamous cell carcinomas of the head and neck includes surgical salvage with reconstruction along with additional therapeutic options such as chemotherapy and radiation. Complete resection with the widest possible margins is recommended and chemo-radiotherapy is added for close or positive margins, for neck disease and for aggressive recurrent high grade tumors. Head and neck cancer is best treated through a multidisciplinary team approach; the most important factor in these cases is very diligent follow up and maintenance of a high index of suspicion for possible recurrence so that the cancer can be caught early and addressed with careful planning and timely individualized patient tailored treatment.

\section{Acknowledgements}

None.

\section{Conflicts of interest}

There is no conflict of Interest.

\section{Funding}

None.

\section{References}

1. Goodwin WJ. Salvage surgery for patients with recurrent squamous cell carcinoma of the upper aerodigestive tract: when do the ends justify the means? Laryngoscope. 2000;110(3 Pt 2 Suppl 93):1-18.

2. Lavertu P, Adelstein DJ, Saxton JP, et al. Aggressive concurrent chemoradiotherapy for squamous cell head and neck cancer. Arch Otolaryngol Head Neck Surg. 1999;125(2):142-148.

3. Wong SJ, Machtay M, Li Y. Locally recurrent, previously irradiated head and neck cancer: concurrent re-irradiation and chemotherapy or chemotherapy alone? J Clin Oncol . 2006;24(17):2653-2658.

4. De Crevoisier R, Domenge C, Wibault P, et al. Full dose re-irradiation combined with chemotherapy after salvage surgery in head and neck carcinoma. Cancer. 2001;91(11):2071-2076.

5. Ken-ichi N. Partial laryngectomy in the treatment of radiation-failure of early glottic carcinoma. Head Neck . 1997;19(2):116-120.

6. Laccourreye O, Weinstein G, Naudo P, et al. Supracricoid partial laryngectomy after failed laryngeal radiation therapy. Laryngoscope. 1996;106(4):495-498.

7. Davidson J, Keane T, Brown D, et al. Surgical salvage after radiotherapy for advanced laryngopharyngeal carcinoma. Arch Otolaryngol Head Neck Surg. 1997;123(4):420-424.

8. Graner DE, Foote RL, Kasperbauer JL, et al. Swallow function in patients before and after intra-arterial chemoradiation. Laryngoscope. 2003;113(3):573-579.

9. Pradhan SA, Rajpal RM, Kothary PM. Surgical management of postradiation residual/recurrent cancer of the base of tongue. J Surg Oncol. 1980;14(3):201-206.

10. Chua DT, Sham JS, Kwong DL, et al. Locally recurrent nasopharyngeal carcinoma: treatment results for patients with computed tomography assessment. Int J Radiat Oncol Biol Phys 41(2): 379-386.

11. Cmelak AJ, Cox RS, Adler JR, et al. Radiosurgery for skull based malignancies and nasopharyngeal carcinoma. Intl J Radiat Oncol Biol Phys. 1997;37(5):997-1003.

12. Buatti J. Linac radiosurgery for locally recurrent nasopharyngeal carcinoma: rationale and technique. Head Neck. 1995;17(1):14-19.

13. Firlik KS, Kondziolka D, Lunsford LD, et al. Radiosurgery for recurrent cranial base cancer arising from the head and neck. Head Neck. 1996;18(2):160-165.

14. Sabri A, Sniezek J, Burkey B. Sensate free flaps. Operative Techniques in Otolaryngology-Head and Neck Surgery. 2000;11(3):195-197.

15. Sabri A. Oropharyngeal reconstruction: current state of the art. Curr Opin Otolaryngol Head Neck Surg. 2003;11(4):251-254.

16. Argiris A, Li Y, Murphy BA, Langer CJ, et al. Outcome of elderly patients with recurrent or metastatic head and neck cancer treated with cisplatin-based chemotherapy. J Clin Oncol. 2004; 22(2):262-228.

17. Bonner JA, Harari PM, Giralt J, et al. Radiotherapy plus cetuximab for squamous-cell carcinoma of the head and neck. $N$ Engl J Med. 2006;354(6):567-578.

18. Wenig B. New Therapies for Locoregionally advanced and Locoregionally Recurrent Head and Neck Cancer. Cancer Treat Res. 2003;114:315-330. 
19. Clayman GL. The Current Status of Gene Therapy. Sem Oncol. 2000;27(4 Suppl 8):39-43.

20. Meyza JW, Towpik E. Surgical and cryosurgical salvage of oral and oropharyngeal cancer recurring after radical radiotherapy. Eur J Surg Onc. 1991;17(6):567-570.

21. Pacheco-Ojeda L, Marandas P, Julieron M, et al. Salvage surgery by composite resection for epidermoid carcinoma of the tonsillar region. Arch Otolaryngol Head Neck Surg. 1992; 118(2):181-184.

22. Pradhan SA, Rajpal RM, Kothary PM. Surgical management of postradiation residual/recurrent cancer of the base of tongue. J Surg Oncol. 1980;14(3):201-206.

23. Clayman GL, Johnson CJ, Morrison W,et al. The role of neck dissection after chemoradiotherapy for oropharyngeal cancer with advanced nodal disease. Arch Otolaryngol Head Neck Surg. 2001;127(2):135-139.

24. Quer M, Leon X, Orus C, et al. Endoscopic laser surgery in the treatment of radiation failure of early laryngeal carcinoma. Head Neck. 2000;22(5):520-523.

25. Shu $\mathrm{CH}$, Cheng H, Lirng JF, et al. Salvage surgery for recurrent nasopharyngeal carcinoma. Laryngoscope. 2000;110(9):1483-1488.

26. King WW, $\mathrm{Ku} \mathrm{PK}$, Mok CO, et al. Nasopharyngectomy in the treatment of recurrent nasopharyngeal carcinoma; a twelve-year experience. Head Neck. 2000;22(3):215-222.

27. Hsu MM, Ko JY, Sheen TS. Salvage surgery for recurrent nasopharyngeal carcinoma. Arch Otolaryngol Head Neck Surg. 1997;123:305-309.

28. Creak AL, Harrington K, Nutting C. Treatment of recurrent head and neck cancer: re-irradiation or chemotherapy? Clin Oncol . 2005;17(3):138-147.

29. Ohizumi Y, Tamai Y, Imamiya S, et al. Prognostic factors of reirradiation for recurrent head and neck cancer. Am J Clin Oncol. 2002;25(4):408-413.

30. Kao J, Garofalo MC, Milano MT, et al. Re-irradiation of recurrent and second primary head and neck malignancies: a comprehensive review. Cancer Treat Rev. 2003;29(1):21-30.

31. Wong SJ, Machtay M, Li Y. Locally recurrent, previously irradiated head and neck cancer: concurrent re-irradiation and chemotherapy or chemotherapy alone? J Clin Oncol. 2006;24(17): 2653-2658.

32. Hwang JM, Fu KK, Phillips TL. Results and prognostic factors in the retreatment of locally recurrent nasopharyngeal carcinoma. Int J Radiat Oncol Biol Phys. 1998;41(5):1099-11111.

33. Hall CE, Harris R, A'Hern R, et al. Le Fort I osteotomy and low-dose rate Ir192 brachytherapy for treatment of recurrent nasopharyngeal tumours. Radiother Oncol. 2003;66(1):41-48.
34. Nieder C, Milas L, Ang KK. Tissue tolerance to re-irradiation. Semin Radiat Oncol. 2000;10(3):200-209.

35. Chua DT, Sham JS, Hung KN, et al. Stereotactic radiosurgery as a salvage treatment for locally persistent and recurrent nasopharyngeal carcinoma. Head Neck. 1999;21(7)|:620-626.

36. Ryu S, Khan M, Yin FF, et al. Image-guided radiosurgery of head and neck cancers. Otolaryngol Head Neck Surg . 2004;130(6):690-697.

37. Pinheiro AD, Foote RL, McCaffrey TV, et al. Intraoperative radiotherapy for head and neck and skull base cancer. Head Neck. 2003;25(3):217-225.

38. Ross DA, Hundal JS, Son YH, et al. Microsurgical free flapl reconstruction outcomes in head and neck cancer patients after surgical extirpation and intraoperative brachytherapyl. Laryngoscope. 2004;114(7):1170-1176.

39. Murphy B, Li Y, Cella D. Phase II study comparing cisplatin and 5-FU versus cisplatin and paclitaxel in metastatic/recurrent head and neck cancer. Proc Am Soc Clin Oncol. 2001;20: 894a.

40. Bendahmane B, Bontemps P, Bourgeois H. Final results of phase II study of patients with metastatic or recurrent squamous cell carcinoma of the head and neck treated with docetaxel and 5-FU. Proc Am Soc Clin Oncol. 2000;19:1656a.

41. Burtness BA, Li Y, Flood W. Phase III trial comparing cisplatin and placebo to cisplatin and anti-EGF antibody C225 in patients with metastatic/recurrent head and neck cancer. Proc Am Soc Clin Oncol. 2002;21:901a.

42. Haraf DJ, Chung K, Stenson M. High dose re-irradiation with concomitant chemotherapy for local/regionally recurrent head and neck cancer (NHC): results in 48 patients. Proc Am Soc Clin Oncol. 2000;19:413a.

43. Hartsell W, Thomas C, Murthy A, et al. Pilot study for the evaluation of simultaneous cisplatin/5-FU infusion and limited radiation therapy in regionally recurrent head and neck cancer. Am J Clin Oncol. 1994;17(4):338-343.

44. Spencer S. Concomitant chemotherapy and re-irradiation as management for recurrent cancer of the head and neck. Am J Clin Oncol. 1994;22(1):1-5.

45. Spencer SA, Harris J, Wheeler RH, et al. RTOG 96-10: re-irradiation with concurrent hydroxyurea and 5-FU in patients with squamous cell cancer of the head and neck. Int $J$ Radiat Oncol Biol Phys. 2001;51(5):1299-1304.

46. Kamar FG, Kairouz VF, Sabri A. Dermatofibrosarcoma protuberans (DFSP) successfully treated with sorafenib: case report. Clin Sarcoma Res. 2013;3(1):5.

47. Cheng LC, Chiu CS, Lee JW. Surgical resection of pulmonary metastases. Journal Cardiovasc Surg. 1998;39(4):503-507. 